\title{
Dynamic model of dynamo (magnetic activity) and rotation
}

\author{
Aditi Sood and Eun-jin Kim
}

\author{
School of Mathematics and Statistics, University of Sheffield, S3 7RH, UK \\ e-mail: [smp11as; e.kim] @sheffield.ac.uk
}

Received 6 August 2012 / Accepted 19 April 2013

\section{ABSTRACT}

\begin{abstract}
A dynamic model of dynamo and rotation is investigated to understand the observational data of the dependence of the magnetic activities and the differential rotation $\Delta \Omega$ on the rotation rate $\Omega$. Specifically, we propose a minimal seventh-order nonlinear dynamical system for magnetic fields and differential rotation $\Delta \Omega$ by parameterizing the generation and destruction of magnetic fields by $\alpha-\Omega$ effect and magnetic flux loss from stars and by including quenching of $\alpha$-effect and differential rotation $\Delta \Omega$ due to the Lorentz force. By examining different forms of $\alpha$-quenching and flux loss, we study how the strength and frequency $\omega$ of magnetic fields and the differential rotation $\Delta \Omega$ change with the rotation rate $\Omega$ through dynamo number. In particular, among the three cases with (i) $\alpha$-quenching and no flux loss; (ii) flux loss and no $\alpha$-quenching; (iii) $\alpha$-quenching and flux loss, our results show that the best agreement with observations is obtained in case (iii) with equal amounts of $\alpha$-quenching and poloidal and toroidal magnetic flux losses with quadratic nonlinear dependence on $|B|$. Specifically, in this case, the frequency spectrum of the magnetic field has a well-localized frequency of the maximum intensity which scales as $\omega \propto \Omega^{0.80}$, in agreement with a previous observation. The magnetic field and mean differential rotation exhibit the tendency of saturation for high rotation. The implication of our results in light of necessary dynamic balance is discussed.
\end{abstract}

Key words. dynamo - magnetohydrodynamics (MHD) - stars: rotation - stars: magnetic field - stars: activity

\section{Introduction}

Magnetic activity occurring in the stellar surface originates through dynamo action, which generates magnetic fields by inductive motion of a conducting fluid in the stellar interior. Different models have been proposed to understand the stellar magnetic activity and the magnetohydrodynamic (MHD) mechanism occurring in stellar interior. A first successful dynamo model was proposed by Parker (1955) which considers the co-action of non-uniform rotation and cyclonic convection. This model generates a toroidal field from poloidal field and a poloidal field from toroidal field, respectively. Since then, mean field models have mainly been used to study magnetic activity, butterfly diagrams, and sunspots by placing various constraints and requirements on fluid motions beneath the stellar surface. The key feature that determines the strength of the magnetic field is the stellar rotation rate. The traditional mean field dynamo theory is based upon the assumption that both $\alpha$-effect and differential rotation, the two key ingredients for mean field dynamo, increase linearly with rotation rate, which is related to a dimensionless dynamo number $D$. As notable previous numerical studies, Gilman (1983a,b) investigated the behavior of nonlinear dynamos with varying $D$ using numerical simulation of MHD equations, whereas Catteneo et al. (1983) and Weiss et al. (1984) studied nonlinear dynamos by using low-order models. Observationally, Noyes et al. (1984) showed the dependence of chromospheric levels and surface magnetic activity in lower main sequence stars on the inverse Rossby number $\sigma=\tau_{\mathrm{c}} / P_{\text {rot }}$, where $\tau_{\mathrm{c}}$ is the convective turnover time and $P_{\text {rot }}$ is the stellar rotation period at the base of the convection zone. In particular, they examined the relationship between magnetic activity and dynamo number $D$. Specifically, they found that for stars of the same spectral type, the cycle period $P_{\text {cyc }}$ of magnetic fields depends upon the stellar rotation period $P_{\text {rot }}$ as $P_{\text {cyc }} \propto P_{\text {rot }}^{\beta}$, with scaling exponent $\beta=1.25 \pm 0.5$. Detailed and systematic investigation of different types of stars has revealed two branches of stars with different scaling exponents, $\beta=0.8$ for active branch and $\beta=1.15$ for inactive branch (Saar \& Brandenburg 1999; Charbonneau \& Saar 2001; Saar 2002).

While differential rotation (shear), one of the crucial ingredient for $\alpha-\Omega$ dynamo, plays a vital role in understanding various activities of stellar magnetism, it has been a subject of investigations on its own for a long time. Starspots are the most visible feature of the magnetic activity occurring as a result of differential rotation (shear) inside the star. Differential rotation (shear) is observed in stellar bodies like stars when the rotation rate varies with latitude. Typically, the increasing latitude causes the decrease in the rotation rate. Observationally, the dependence of differential rotation on rotation is quite uncertain. Various studies that have investigated the relationship between stellar angular rotation $\Omega$ and its latitudinal difference $\Delta \Omega$, that is, $\Delta \Omega \propto \Omega^{n}$ suggested a broad range of $n$ as $0<n<1$ (Fröhlich et al. 2009; Hotta \& Yokoyama 2011; Donahue et al. 1996; Reiners \& Schmitt 2003; Barnes et al. 2005). This suggests that the angular velocity difference $\Delta \Omega$ increases and the relative difference $\Delta \Omega / \Omega$ decreases with increase in the stellar rotation rate $\Omega$. For instance, Barnes et al. (2005) showed that in cool stars differential rotation $\Delta \Omega$ virtually does not depend on rotation rate as $\Delta \Omega \propto \Omega^{0.15}$, whereas Saar (2011) showed a rather different result between differential rotation and rotation rate as $\Delta \Omega \propto \Omega^{0.68}$ that is very similar to HK-based results of $\Delta \Omega \propto \Omega^{0.70}$. Furthermore, time variation of differential rotation has been reported in recent work (e.g., Fröhlich et al. 2009; Hotta \& Yokoyama 2011).

In $\alpha-\Omega$ mean field dynamo, mean helicity thought to be crucial for $\alpha$ - effect is produced due to rotation (Moffat 1978; Parker 1979). The regeneration of poloidal magnetic field thus depends on a parameter $\alpha=-\frac{\tau_{\mathrm{c}}}{3}\langle u(\nabla \times u)\rangle$, where $u$ is the convective velocity with magnitude $U$ and $\tau_{\mathrm{c}}=L / U$ is the convective turnover 
time on characteristic length scale $L$. The $\alpha$-effect can thus be measured by a non-dimensional parameter $D_{\alpha}=\alpha L / \eta$, where $\eta=L^{2} / \tau_{\mathrm{c}}$ is turbulent magnetic diffusivity. On the other hand, the efficiency of the generation of a toroidal magnetic field due to differential rotation (shear) is measured by a magnetic Reynold number $D_{\Omega}=\Omega^{\prime} L^{3} / \eta$, where $\Omega^{\prime} \propto \Omega / L$ is the gradient of rotation rate $\Omega$. The dimensionless dynamo number $D$ governing dynamo action is thus given by

$D=D_{\alpha} D_{\Omega}=\frac{\alpha L}{\eta} \frac{\Omega^{\prime} L^{3}}{\eta}$

By assuming $\alpha$ is proportional to rotation rate, we then obtain the dependence of $D$ on $\Omega$ as

$D \propto \Omega^{2}$.

For stars with the same internal structure, the cycle period $P_{\text {cyc }}$ of the magnetic field is related to the rotation rate $\Omega$ as $P_{\text {cyc }} \propto$ $D^{-\frac{1}{2}} \propto \Omega^{-1}$ in the case of linear dynamo theory so that frequency of magnetic fields scales with $\Omega$ as $\omega \propto P_{\text {cyc }}^{-1} \propto \Omega$, that is, the period of the magnetic field decreases with rotation rate, which is compatible with observations. However, in nonlinear dynamo, the growth of the magnetic field is limited by dynamo saturation, and it is thus not at all clear if this linear relation still holds. For instance, there are at least three different saturation mechanisms that have been proposed for stellar dynamo theory: (i) $\alpha$-quenching: the quenching of helicity by the magnetic field, causing the growth of the mean field to be saturated; that is, large magnetic field strength reduces the total helicity and dynamo action is ceased; (ii) $\Omega$-quenching ( shear quenching): mean differential rotation is opposed by the tension in the magnetic field lines by the Lorentz force, reducing $\Omega$-effect; (iii) magnetic flux loss: the growth of the magnetic field can be inhibited by the magnetic flux removed from the region in which the dynamo operates; for instance, magnetized fluid lighter than the unmagnified surroundings becomes buoyant as reduced magnetic pressure inside the tube along with density makes it rise against the gravity due to buoyancy. In particular, the first two saturation mechanisms ( $\alpha$ and $\Omega$-quenching) seriously question the validity of the classical $\alpha$ - $\Omega$ dynamo (e.g., Charbonneau 2005, and references therein). Furthermore, in the previous work, the models in which the magnetic field is limited by the quenching of $\alpha$-effect or of differential rotation (Jepps 1975; Ivanova \& Ruzmaikin 1977; Yoshimura 1978) were shown to be in disagreement with observations of stellar rotation and activity cycle period. While there is no consensus on a precise form of the stellar/solar dynamo, the advection-dominated dynamo (e.g., Dikpati \& Charbonneau 1999) has been the most popular model for explaining several features of the solar activity cycle.

The purpose of this paper is to use the aforementioned observational data, specifically, the dependence of the frequency of magnetic field and its strength (inferred from magnetic activity) and differential rotation on stellar rotation rate, to identify the key feature of the dynamo process, which is required for a successful dynamo model. To this end, we propose a minimal dynamical model for magnetic fields and differential rotation and perform a detailed analysis by varying various parameters to constrain the model to reproduce results consistent with observational data. Specifically, we implement $\alpha$-quenching, magnetic flux loss, and the Lorentz force on mean and fluctuating differential rotations in a low-order dynamo model of seven coupled equations for magnetic fields and differential rotations, and compare our results with observations. Principle results are that the observed relation between magnetic frequency and rotation rate results from a nonlinear balance between the generation and destruction of the magnetic fields, which makes the dynamo operate close to stability. This leads to the almost linear increase in frequency with rotation rate with a rather well-localized frequency (reminiscent of a linear dynamo), flattening of magnetic energy for high rotation, and quenching in total shear consistent with observation. Furthermore, we suggest that the long-range correlation in stars and open boundaries could be a key feature necessary to reproduce observation.

We admit the limitations of our model, which is, however, likely to be more valid for slow rotators in inactive branch than fast rotators. Whether our model is applicable to fast rotating stars seems to depend on the identification of fundamental physical mechanisms causing these two distinct branches. It is not inconceivable that fast rotation and/or strong differential rotation introduce additional physics (e.g., quenching of eddy viscosity, Kichatinov 2012) that may have to be taken into account to explain these two branches. Moreover, we should note that Saar (2002) was inconclusive as to the most promising models that best fit the data of these two branches. In particular, he noted that the $\alpha-\Omega$ dynamo, where differential rotation and $\alpha$-effect strongly depend upon rotation rate, could explain these two branches (Saar 2002).

The remainder of the paper is organized as follows. Section 2 presents our extended dynamo model. Section 3 contains the results of seventh-order system. In Sect. 4 we study the system in the limit where mean differential rotation dominates fluctuating differential rotation while in Sect. 5 we study the system in the opposite limit. Section 6 elaborates on the basic property of the seventh-order system in Sect. 2, in particular, in light of the balance between all nonlinearities and investigate a more general seventh-order system to check on parameter dependencies. In Sect. 7, we discuss our results and conclude.

\section{Model construction}

By taking into account dynamical interactions between the magnetic field and differential rotation, we build upon a simple parameterized dynamo model that was constructed by Cattaneo et al. (1983) based on $\alpha-\Omega$ dynamo. Specifically, plane wave solutions propagating in $x$-direction (Parker 1979), in the presence of azimuthal velocity $v=(V(z)+W(x, z, t)) \hat{y}$, which consist of rotation velocity $V=\Omega z$ (where $z$ is the radial coordinate) and the differential rotation $W(x, z, t)$ due to back reaction, were considered taking local cartesian co-ordinates $y$-axis and $z$-axis pointing westward and radially outward, respectively. Periodic boundary conditions are assumed for the model in terms of Fourier modes and for a magnetic field of $k$-mode $B=\left(0, B(t) \mathrm{e}^{\mathrm{i} k x}, \mathrm{i} k A(t) \mathrm{e}^{\mathrm{i} k x}\right)$. The Lorentz force generates the differential rotation $\frac{\partial W}{\partial z}=w_{0}+w(t) \exp (2 \mathrm{i} k x)$, where $w_{0}$ and $w(t)$ are mean and fluctuating differential rotation, respectively, leading to a low-order dynamo model given by seventhorder coupled equations in the following dimensionless forms:

$$
\begin{aligned}
\dot{A} & =2 D B-A, \\
\dot{B} & =\mathrm{i}\left(1+w_{0}\right) A-\frac{1}{2} \mathrm{i} A^{*} w-B, \\
\dot{w_{0}} & =\frac{1}{2} \mathrm{i}\left(A^{*} B-A B^{*}\right)-v_{0} w_{0}, \\
\dot{w} & =-\mathrm{i} A B-v w .
\end{aligned}
$$

Here $A$ is the poloidal magnetic flux, $B$ is the toroidal magnetic field, and $w$ represents fluctuating differential rotation with twice the frequency of poloidal and toroidal magnetic fields. While $A$, $B$, and $w$ are complex, $w_{0}$ is the mean component of differential 


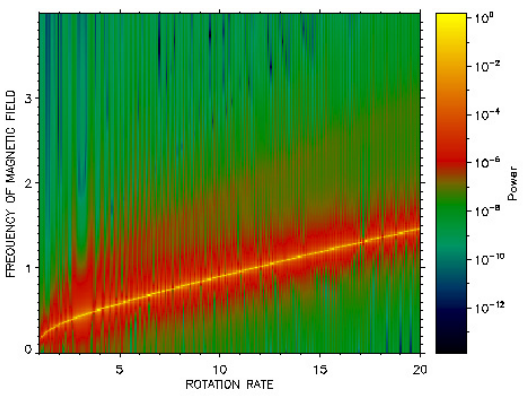

(a) $\omega_{\mathrm{M}}$ as a function of $\Omega$

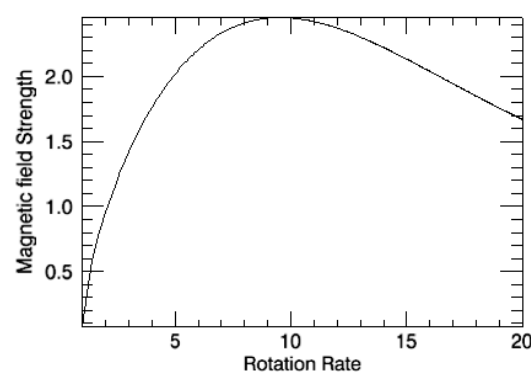

(b) $|B|$ as a function of $\Omega$

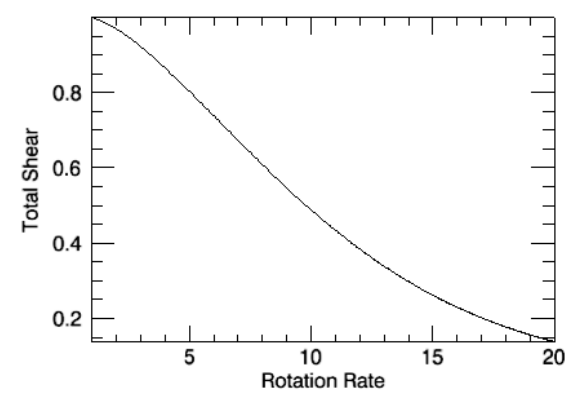

(c) Total shear as a function of $\Omega$

Fig. 1. Frequency of maximum intensity $\omega_{\mathrm{M}}$, magnetic field strength $|B|$ and total shear are plotted as a function of $\Omega$ for $\kappa_{1}=2.5, \lambda_{1}=\lambda_{2}=0$ for Case 1 in the seventh-order system.

rotation, which is real. We note that in these dimensionless units the total mean differential rotation is represented by $1+w_{\circ}=\frac{\Delta \Omega}{\Omega}$, whose numerical value is always less than unity as $w_{\circ}$ is a negative constant. In addition, $v$ and $v_{0}$ are constants representing viscosity of mean and fluctuating differential rotations, respectively, and $D$ is the dynamo number. We note that Eqs. (3)-(6) possess the same linear dispersion relation $\omega=k^{2}\left[-1 \pm(1+i) D^{1 / 2}\right]$ as given by Weiss (1984) and that leads to finite amplitude nonlinear solutions for $D>1$. We extend the above system (3)-(6) by taking into account $\alpha$-quenching and magnetic flux loss as follows:

$$
\begin{aligned}
\dot{A} & =\frac{2 D B}{1+\kappa_{1}\left(|B|^{2}\right)}-\left[1+\lambda_{1}\left(|B|^{2}\right)\right] A, \\
\dot{B} & =\mathrm{i}\left(1+w_{0}\right) A-\frac{1}{2} \mathrm{i} A^{*} w-\left[1+\lambda_{2}\left(|B|^{2}\right)\right] B, \\
\dot{w}_{0} & =\frac{1}{2} \mathrm{i}\left(A^{*} B-A B^{*}\right)-v_{0} w_{0} . \\
\dot{w} & =-\mathrm{i} A B-v w .
\end{aligned}
$$

Here, $\lambda_{1}\left(|B|^{2}\right), \lambda_{2}\left(|B|^{2}\right)$, and $\kappa_{1}\left(|B|^{2}\right)$ are assumed to be functions of $|B|$ to include the feedback of growing magnetic field onto itself: $\lambda_{1}\left(|B|^{2}\right)$ captures the effect on the generation of the magnetic field, while $\lambda_{2}\left(|B|^{2}\right)$ and $\kappa_{1}\left(|B|^{2}\right)$ capture the effect on the dissipation of magnetic field. All three terms become zero when $B=0$ and are assumed to increase with $|B|$ (Robinson \& Durnery 1982; Weiss et al. 1984) to model $\alpha$-quenching and enhanced magnetic dissipation for strong magnetic field. We note that the latter is in contrast to some other previous work using the quenching of magnetic dissipation (e.g., Brandenberg et al. 2008). For our minimal model, we consider $\lambda_{1}\left(|B|^{2}\right)=\lambda_{1}|B|^{2}$, $\lambda_{2}\left(|B|^{2}\right)=\lambda_{2}|B|^{2}$, and $\kappa_{1}\left(|B|^{2}\right)=\kappa_{1}|B|^{2}$ with constant coefficients of $\lambda_{1}, \lambda_{2}$, and $\kappa_{1}$. Thus, non-zero $\kappa_{1}$ represents the nonlinear $\alpha$-effect quenching while non-zero $\lambda_{1}$ and $\lambda_{2}$ represent loss of poloidal and toroidal fields due to magnetic flux loss, respectively. In our extended model, $\alpha$ simply parameterizes the efficiency of the generation of poloidal magnetic field from toroidal field (for instance, by helicity) which is assumed to linearly increase with rotation rate. We note that similar quadratic $\alpha$-quenching and flux loss were used previously, but in a simpler, fourth-order system without differential rotation (e.g., Weiss et al. 1984).

\section{Seventh-order system}

We investigate our extended model (7) to (10) for the following three cases by taking $v=1.0$ and $v_{0}=35.0$ and by varying $D$ from 1 to 400.

Case 1: $\alpha$-quenching and no flux loss i.e., $\lambda_{1}=\lambda_{2}=0, \kappa_{1} \neq 0$
Case 2: no $\alpha$-quenching and flux loss i.e., $\lambda_{1}=\lambda_{2}, \kappa_{1}=0$ Case 3: $\alpha$-quenching and flux loss i.e., $\lambda_{1}=\lambda_{2}=\kappa_{1}$.

We solve Eqs. (7) to (10) numerically and study how the frequency of magnetic activity $\omega$ varies with $D$ and $\Omega\left(\Omega=D^{\frac{1}{2}}\right.$ as noted in the introduction). To obtain the frequency of maximum intensity for the magnetic activity of each dynamo number, we obtain a time series of all the dynamical variables (say $B$ ) and use fast Fourier transforms to obtain a Fourier series of $B$ to compute the power spectrum of frequency for each dynamo number. Once we have the power spectra for all dynamo numbers, we assign the color according to the color coding as shown in the bar next to the figure, where yellow to dark colors denote frequency of maximum intensity to low intensity. We also study the variation of magnetic field strength $|B|$ and differential rotation $\Delta \Omega$ with rotation rate $\Omega$. We note that total shear is related to rotation rate $\Omega$ using the power law as $1+w_{\circ}=\Omega^{\delta}$, where $\delta$ is the scaling exponent. Normalized differential rotation is given as $1+w_{\circ}=\Delta \Omega / \Omega$.

Case 1: $\alpha$-quenching is causing the saturation (i.e., $\lambda_{1}=\lambda_{2}=0$, $\left.\kappa_{1}=2.5\right)$. We examine how frequency, say $\omega_{\mathrm{M}}$, of $B$ of maximum intensity varies with $\Omega$ by using the power law as $\omega_{M}=\Omega^{\beta}$, where $\beta$ is the scaling exponent (cf. Fig. 1a). We also study how $|B|$ and total shear change with rotation rate (Figs. 1b, c). In Fig. 1a, we plot the frequency of $B$ as a function of rotation rate, where yellow to dark black colors represent the high to low intensity of the frequency. It is interesting to see that there is a well-defined frequency of maximum intensity $\omega_{\mathrm{M}}$ denoted in yellow, which slowly increases with rotation rate $\Omega$. The dependence of frequency of maximum intensity $\omega_{M}$ on $\Omega$ is found to be a power law using $\omega_{\mathrm{M}}=\Omega^{\beta}$ with the two scaling exponents of $\beta=0.67$ and 1.24 for slow and high rotations, respectively. This is not in agreement with observation. The band in red around $\omega_{\mathrm{M}}$ represents the dispersion in the frequency, which slowly reduces its width with increasing $\Omega$. Figure $1 \mathrm{~b}$ shows the strength of toroidal magnetic field $|B|$ as a function of $\Omega$. It is clearly seen that $|B|$ increases up to $\Omega \sim 6$ with increasing $\Omega$ before decreasing for $\Omega \geq 10$. The decrease of $|B|$ for $\Omega>10$ is caused by the quenching of mean differential rotation $w_{0}$ due to the Lorentz force of magnetic fields. We show this shear quenching in Fig. 1c, where total shear $1+w_{\circ}$ is plotted as a function of $\Omega$. This total shear becomes very small as high rotation and does not exhibit a reasonable power-law scaling.

Case 2: magnetic flux loss alone leads to saturation (i.e., $\left.\lambda_{1}=\lambda_{2}=2.5, \kappa_{1}=0.0\right)$. We again examine the behaviors of $\omega_{\mathrm{M}}$, the magnetic field strength $|B|$, and the total shear for different rotation rates (cf. Figs. $2 a-c$ ). In Fig. 2 a, the frequency 


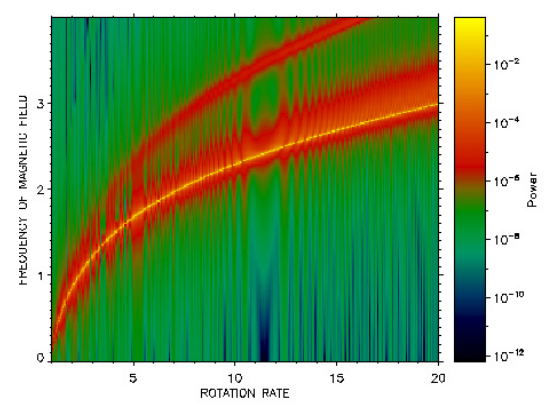

(a) $\omega_{\mathrm{M}}$ as a function of $\Omega$

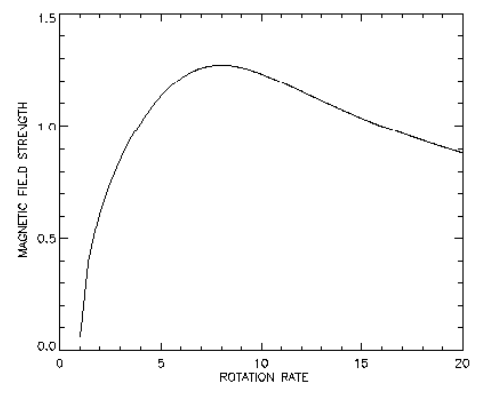

(b) $|B|$ as a function of $\Omega$

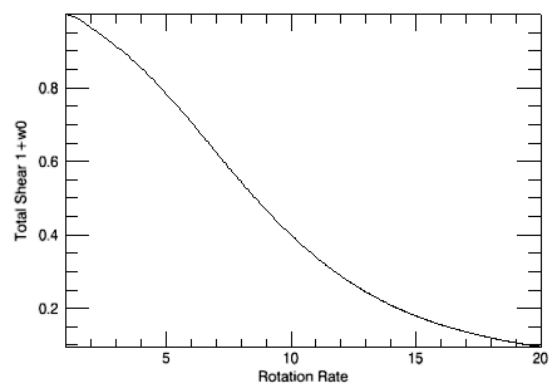

(c) Total shear as a function of $\Omega$

Fig. 2. Frequency of maximum intensity $\omega_{\mathrm{M}}$, magnetic field strength $|B|$, and total shear are plotted as a function of $\Omega$ for $\kappa_{1}=0.0, \lambda_{1}=\lambda_{2}=2.5$ for Case 2 in the seventh-order system.

of $B$ is plotted as a function of rotation rate. Here, yellow color again indicates the frequency of high intensity and dark black colors indicate the frequency of low intensity. A main branch of maximum intensity with a well-defined frequency $\omega_{M}$ denoted in yellow increases with rotation rate $\Omega$ at a rate faster than in Fig. 1a. We use the power law $\omega_{\mathrm{M}}=\Omega^{\beta}$ to examine the dependence of frequency of maximum intensity $\omega_{\mathrm{M}}$ on $\Omega$, finding that the scaling exponent $\beta$ decreases its value from $\beta=1.08$ to 0.73 for slow-to-high rotation rate. We find that $\beta=1.08$ for $\Omega \leq 7$ and $\beta=0.87$ for $7<\Omega<10$ are compatible with the observed range of $\beta \sim 0.80$ to $\beta \sim 1.15$ whereas $\beta=0.73$ for $\Omega>10$ is close to the observed range. Dispersion in frequency around $\omega_{M}$ broadens with increasing $\Omega$. Interestingly, a second branch of frequency of localized maximum intensity is observed above the main branch, which increases quickly with increasing rotation rate. This second branch arises from fluctuating differential rotation. Figure $2 \mathrm{~b}$ shows the strength of the toroidal magnetic field $|B|$ as a function of $\Omega$. Clearly, $|B|$ increases with $\Omega$ for the stars having a rotation period of five days or longer $(\Omega \leq 6)$ but starts decreasing for faster rotating stars with the a rotation period less than five days, for $\Omega \geq 6$. We note that this decrease in $|B|$ is not as fast observed in Case 1 (cf. Fig. 1b). Here again, mean differential rotation $w_{0}$ due to the Lorentz force causes the decrease of $|B|$ for $\Omega>6$, as can be seen in Fig. 2c.

Case 3: saturation is taking place because of the combined action of $\alpha$-quenching and loss due to magnetic flux (i.e. $\lambda_{1}=\lambda_{2}=$ $\left.\kappa_{1}=2.5\right)$. In Fig. $3 \mathrm{a}$, the frequency spectrum of the magnetic field $B$ as a function of rotation rate shows a well-defined frequency of maximum intensity and a frequency of low intensity, which are depicted in yellow and dark black colors, respectively. In this case, the frequency of maximum intensity $\omega_{M}$ slowly increases with rotation rate $\Omega$. The dependence of the frequency of maximum intensity $\omega_{\mathrm{M}}$ on $\Omega$ is found to exhibit a clear power law scaling in the entire range of $\Omega$ as $\omega_{\mathrm{M}}=\Omega^{\beta}$ with the scaling exponent of $\beta=0.80$ (see Fig. 3d for scaling). This value is the same as that of the observed value of $\beta \sim 0.80$ for active stars. We observe dispersion in the frequency around the well-defined frequency of maximum intensity $\omega_{\mathrm{M}}$, which is depicted in the red band. The width of the band expands moderately with increasing $\Omega$, being broader than the bands observed in Case 1 and Case 2. In Fig. 3b, the strength of the toroidal magnetic field $|B|$ is studied as function of $\Omega$. Interestingly, $|B|$ approaches the asymptotic value of $|B|=1.25$ with an increasing rotation rate $\Omega$. The shear-quenching can be seen in Fig. 3c, where total shear decreases with increasing rotation, but at a much slower rate compared to Case 1 and Case 2.
In summary, among the three cases considered in this seventh-order system, Case 1 and Case 2 with only $\alpha$-quenching or flux loss show the behavior of frequency and strength of magnetic fields that are in disagreement with observation. We observe in both cases that the absence of $\alpha$-quenching or flux loss causes a magnetic field that is too strong for high rotation, which then reduces total shear to a small value by the Lorentz force back-reaction. This feedback therefore makes the dynamo less efficient. Agreement with observations is obtained only in Case 3, which exhibits an equal amount of $\alpha$-quenching and poloidal and toroidal magnetic flux losses. Specifically, in this case, the frequency spectrum of the magnetic field has a well-localized frequency of the maximum intensity which scales as $\omega \propto \Omega^{0.80}$, in agreement with the observation of Noyes et al. (1984). The magnetic field and mean differential rotation exhibit a tendency of saturation for high rotation. These results thus suggest that there must be an effective balance between generation and dissipation of magnetic fields to obtain the saturation of magnetic fields at high rotation. This is explained in detail in Sect. 6.

\section{Reduced fifth-order system}

In the previous section, a too strong mean differential rotation was shown to make dynamo action ineffective. To highlight this and to elucidate the role of the mean differential rotation $w_{0}$ in a mean field dynamo, we consider the extreme limit where fluctuating differential rotation $w$ is much weaker than $w_{0}$ by taking the limits of $v \rightarrow \infty$ and $w \rightarrow 0$ in Eqs. (7) to (10). A reduced fifth-order system in the presence of nonlinearities such as $\alpha$-quenching and magnetic flux loss is then given as follows:

$$
\begin{aligned}
\dot{A} & =\frac{2 D B}{1+\kappa_{1}\left(|B|^{2}\right)}-\left[1+\lambda_{1}\left(|B|^{2}\right)\right] A, \\
\dot{B} & =\mathrm{i}\left(1+w_{0}\right) A-\left[1+\lambda_{2}\left(|B|^{2}\right)\right] B, \\
\dot{w}_{0} & =\frac{1}{2} \mathrm{i}\left(A^{*} B-A B^{*}\right)-v_{0} w_{0} .
\end{aligned}
$$

We examine this fifth-order system with $v_{0}=35.0$ by varying $D$ for the following different three cases;

Case 1: $\kappa_{1} \neq 0, \lambda_{1}=\lambda_{2}=0$,

Case 2: $\kappa_{1}=0, \lambda_{1}=\lambda_{2} \neq 0$,

Case 3: $\kappa_{1}=\lambda_{1}=\lambda_{2} \neq 0$.

Case 1: saturation occurs through quenching of $\alpha$-effect only (i.e., $\lambda_{1}=\lambda_{2}=0, \kappa_{1}=1.0$ ). We examine the behavior of frequency of the magnetic field $\omega_{\mathrm{M}}$, the magnetic field strength $|B|$ 
Aditi Sood and Eun-jin Kim: Dynamic model of dynamo (magnetic activity) and rotation

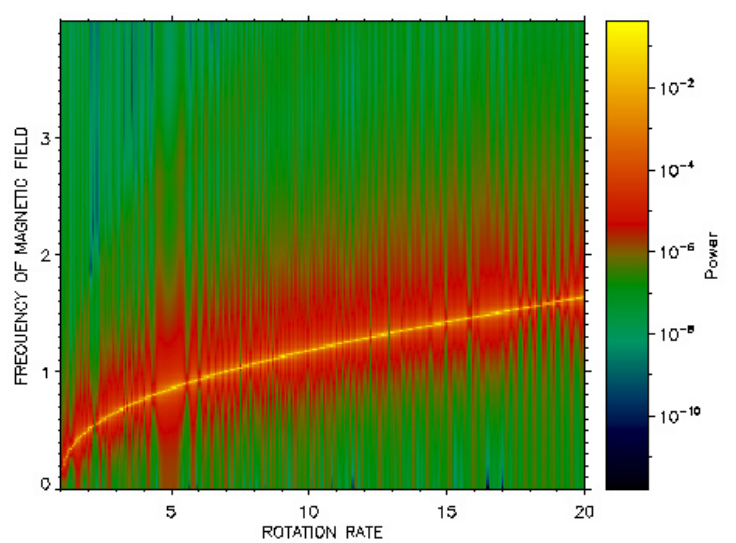

(a) $\omega_{\mathrm{M}}=\Omega^{0.80}$

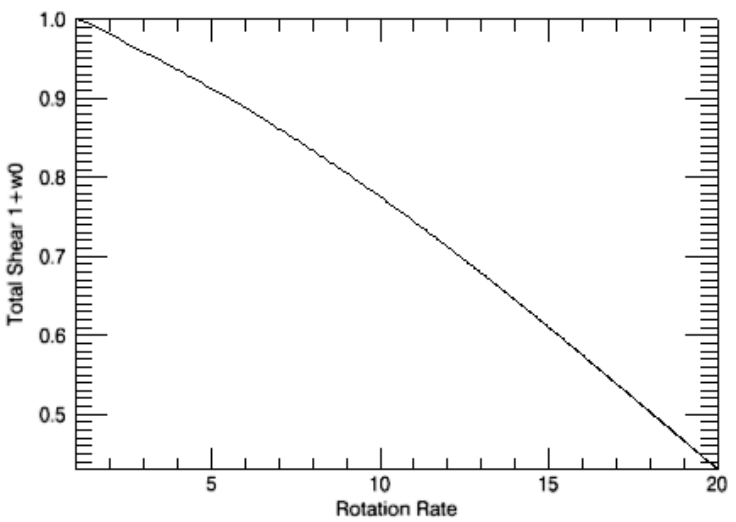

(c) Total shear as a function of $\Omega$

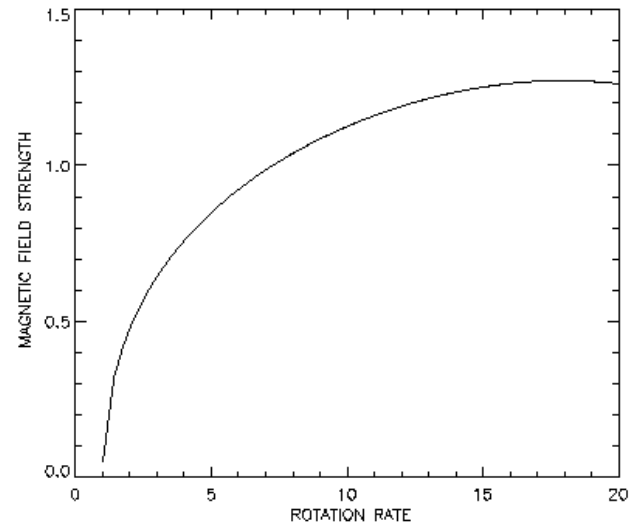

(b) $|B|$ as a function of $\Omega$

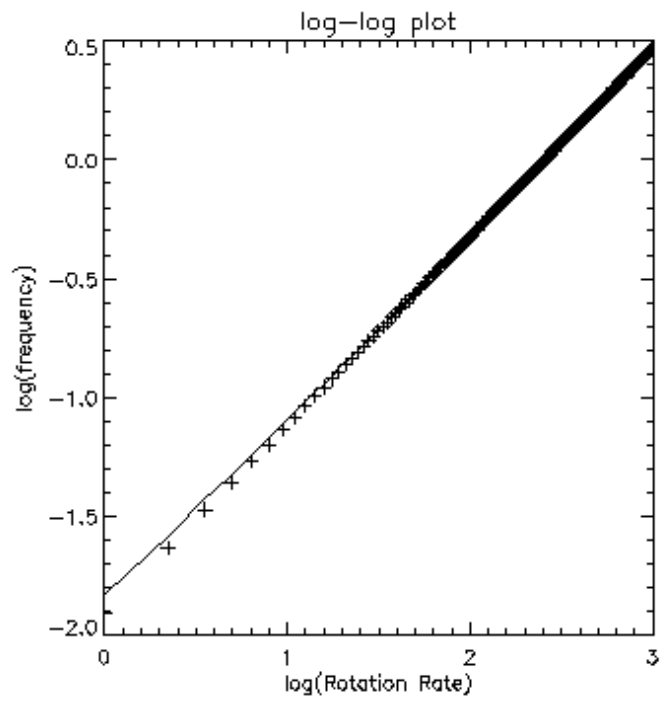

(d) Log-log plot of $\omega_{\mathrm{M}}$ with rotation rate, where curve fitting is represented with '+' symbol.

Fig. 3. Frequency of maximum intensity $\omega_{\mathrm{M}}$, magnetic field strength $|B|$, and total shear are plotted as a function of $\Omega$ for $\kappa_{1}=\lambda_{1}=\lambda_{2}=2.5$ for Case 3 in the seventh-order system.

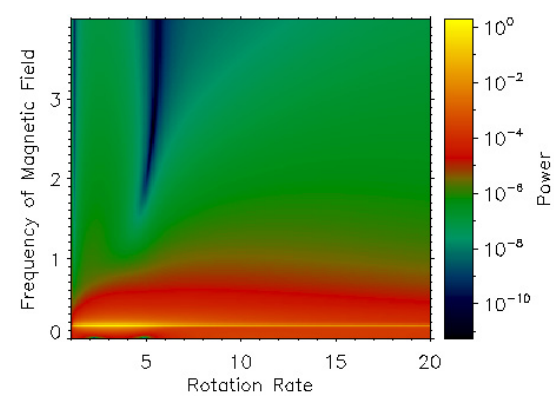

(a) $\omega_{\mathrm{M}}$ as a function of $\Omega$

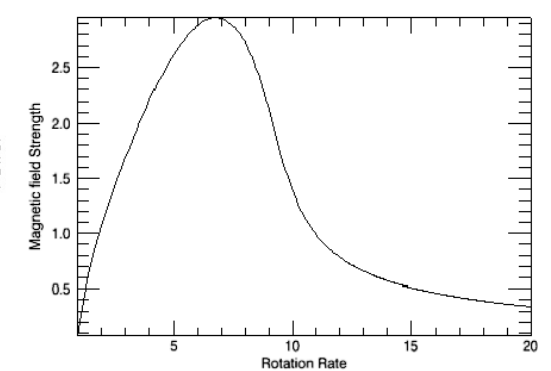

(b) $|B|$ as a function of $\Omega$

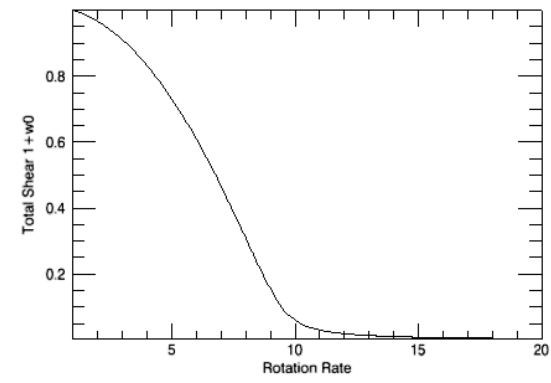

(c) Total shear as a function of $\Omega$

Fig. 4. $\omega_{\mathrm{M}},|B|$, and total shear are plotted as a function of $\Omega$ for $\lambda_{1}=\lambda_{2}=0, \kappa_{1}=1.0$ for Case 1 in the fifth-order system.

(Figs. 4a, b) and the total shear as a function of rotation rate $\Omega$ (Fig. 4c). In Fig. 4a, we plot the frequency spectrum of $B$ as function of the rotation rate where high and low intensity of frequency is again represented by yellow and dark black, respectively. We find a finite amplitude wave with the fixed frequency $\omega_{M}$ of maximum intensity, denoted in yellow, which does not change with rotation, in sharp contrast to observations. Figure $4 \mathrm{~b}$ shows the strength of the magnetic field $|B|$ as a function of $\Omega$. Strength of magnetic activity increases initially with rotation rate up to $\Omega \sim 6$ but starts decreasing for stars with a rotation period of less than five days. These results are due to severe quenching in both $\alpha$-effect and $\Omega$-effect, which makes $D$ almost independent of $\Omega$. Shear quenching can be seen in Fig. 4c, which decreases very rapidly up to a rotation rate $\Omega \sim 10$ and becomes almost zero for $\Omega>10$. It is interesting to note that similar severe quenching of differential rotation and dynamo was also obtained in full MHD simulations using a flux-transport dynamo model (e.g., Rempel 2006). 


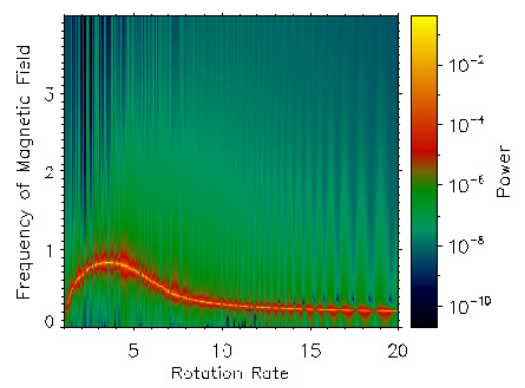

(a) $\omega_{M}$ as a function of $\Omega$

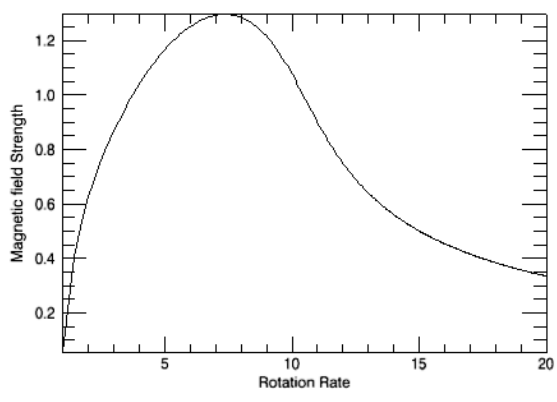

(b) $|B|$ as a function of $\Omega$

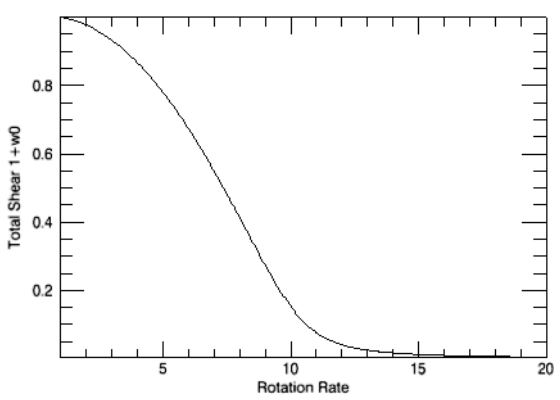

(c) Total shear as a function of $\Omega$

Fig. 5. $\omega_{\mathrm{M}},|B|$, and total shear are plotted as a function of $\Omega$ for $\lambda_{1}=\lambda_{2}=2.5, \kappa_{1}=0.0$ for Case 2 in the fifth-order system.

Case 2: dynamo action is ceased by loss due to magnetic flux only (i.e., $\lambda_{1}=\lambda_{2} \neq 0, \kappa_{1}=0$ ). The behavior of $\omega_{\mathrm{M}},|B|$, and total shear with $\Omega$ is examined (cf. Figs. 5a-c). In Fig. 5a, we explore the frequency spectrum of the magnetic field $B$ as a function of rotation rate. The frequency of maximum intensity denoted by $\omega_{\mathrm{M}}$, represented in yellow, increases with rotation rate up to $\Omega \sim 5$ and then starts decreasing with rotation (cf. Fig. 5a), in strong disagreement with observations. The band of frequency of lower intensity around $\omega_{\mathrm{M}}$, represented in red, again shows the frequency dispersion in the system. The width of band decreases with increasing rotation rate. The dependence of $\omega_{\mathrm{M}}$ on $\Omega$ is found to be a power law as $\omega_{\mathrm{M}}=\Omega^{\beta}$ with a scaling exponent of $\beta=0.91$ for stars with rotation period of six or more days, which is in the observed range of 0.80 to 1.15 . However, this relation breaks down for stars with a rotation period of less than six days. Figure 5b shows that the strength of magnetic activity $|B|$ attains its maximum value for rotation rate $\Omega=7$ and starts decreasing for $\Omega \sim 8$. The decrease of $|B|$ for $\Omega \geq 8$ is caused by the quenching of the mean differential rotation $w_{0}$, as can be seen from Fig. $5 \mathrm{c}$. We find the total shear decreases by up to $\Omega \sim 12$, which is not as fast as noted in Fig. 4c. For $\Omega \geq 12$, the value of shear approaches zero.

Case 3: saturation of dynamo action is caused by the equal combination of $\alpha$-quenching and magnetic flux loss (i.e., $\kappa_{1}=\lambda_{1}=$ $\left.\lambda_{2}=3.5\right)$. The frequency spectrum of $B$ as a function of rotation rate $\Omega$ is shown in Fig. 6a, illustrating high-intensity frequency in yellow and low-intensity frequency in dark black. In comparison to Cases 1 and $2,|B|$ does not become sufficiently strong to dramatically reduce $\Omega$ without causing a dramatic reduction in $D$. Therefore, there is a very slow increase in the frequency of maximum intensity $\omega_{\mathrm{M}}$ with rotation rate $\Omega$, although at a variable rate with the two scaling exponents $\beta=0.63$ for $\Omega \leq 10$ and $\beta=0.45$ for $\Omega>10$. This variation is not in agreement with the observation. The band in red around $\omega_{M}$ represents the dispersion in the frequency, which gradually widens with increasing $\Omega$. Clearly, strength of magnetic field $|B|$ increases slowly with increasing rotation as shown in Fig. 6b, in disagreement with observations. Total shear as function of rotation rate is plotted in Fig. 6c, which shows a rapid decrease in total shear with increasing rotation rate.

In summary, the fifth-order system is not compatible with observations in all possible cases, with a tendency toward too severe quenching of differential rotation and a lower frequency of $B$ than observed. These results imply that fluctuating differential rotation can be important to obtain magnetic field intensity and frequency spectrum consistent with observations (as found in the seventh-order system). It is thus useful to identify the role of fluctuating differential rotation, which is done in the following section.

\section{Reduced sixth-order system}

We now examine the effect of fluctuating differential rotation $w$ by taking the opposite limit to Sect. 4 . That is, the system (7)-(10) is reduced to a sixth-order system by taking $v_{0} \rightarrow \infty$ and $w_{0} \rightarrow 0$. A reduced sixth-order dynamical system in the presence of nonlinearities such as $\alpha$-quenching and magnetic flux loss is given as follows:

$$
\begin{aligned}
& \dot{A}=\frac{2 D B}{1+\kappa_{1}\left(|B|^{2}\right)}-\left[1+\lambda_{1}\left(|B|^{2}\right)\right] A, \\
& \dot{B}=\frac{\mathrm{i} A}{1+\kappa_{2}\left(|B|^{2}\right)}-\frac{1}{2} \mathrm{i} A^{*} w-\left[1+\lambda_{2}\left(|B|^{2}\right)\right] B, \\
& \dot{w}=-\mathrm{i} A B-v w .
\end{aligned}
$$

We note that unlike the seventh- and fifth-order systems, we include the reduction of $\Omega$ effect due to shear quenching by including $1+\kappa_{2}|B|^{2}$ in Eq. (8). We investigate the system (14)-(16) for the following two cases, taking $v=1.0$ and varying $D$ :

Case 1: $\lambda_{1}=\lambda_{2}=\kappa_{1}=\kappa_{2} \neq 0$,

Case 2: $\kappa_{1}=0, \kappa_{2}=\lambda_{1}=\lambda_{2} \neq 0$.

We note that (14) to (16) are reduced to Weiss model (1984) when $\lambda_{1}=\lambda_{2}=\kappa_{1}=\kappa_{2}=0$.

Case 1: dynamo action is saturated by an equal combination of $\alpha$-quenching, shear quenching, and loss due to magnetic flux (i.e., for $\lambda_{1}=\lambda_{2}=\kappa_{1}=\kappa_{2}=0.5$ ). In Fig. 7a, the frequency of $B$ is plotted as a function of rotation rate $\Omega$, depicting high-intensity frequency in yellow and low-intensity frequency in dark black. Here, again, we find a well-defined frequency of maximum intensity $\omega_{\mathrm{M}}$ denoted in yellow, which increases with rotation rate $\Omega$. We use the power law $\omega_{\mathrm{M}}=\Omega^{\beta}$ to study the dependence of $\omega_{\mathrm{M}}$ on rotation rate $\omega$; the scaling exponent $\beta$ is found to be 0.90 , which is in the observed range between $\beta=0.80$ and $\beta=1.15$. Dispersion in the frequency is represented by the red band around $\omega_{\mathrm{M}}$, which expands its width with increasing rotation rate. Figure $7 \mathrm{~b}$ shows that the strength of toroidal magnetic field $|B|$ increases with increasing rotation rate. We find an almost similar trend of $\omega_{\mathrm{M}}$ and $|B|$ when we investigate the system by taking $\lambda_{1}=0, \lambda_{2}=\kappa_{1}=\kappa_{2} \neq 0$, $\lambda_{2}=0, \lambda_{1}=\kappa_{1}=\kappa_{2} \neq 0, \lambda_{1}=\lambda_{2}=0, \kappa_{1}=\kappa_{2} \neq 0$, and $\kappa_{2}=0, \lambda_{1}=\lambda_{2}=\kappa_{1} \neq 0$. 
Aditi Sood and Eun-jin Kim: Dynamic model of dynamo (magnetic activity) and rotation

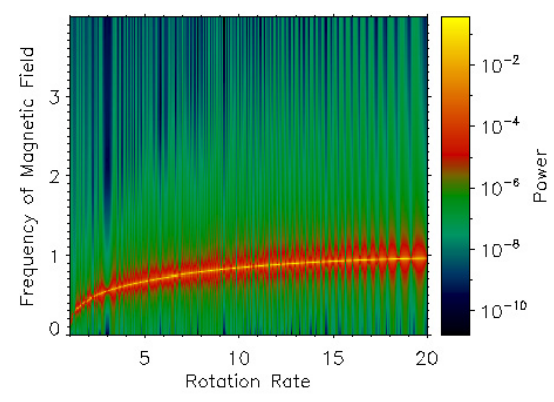

(a) $\omega_{\mathrm{M}}$ as a function of $\Omega$

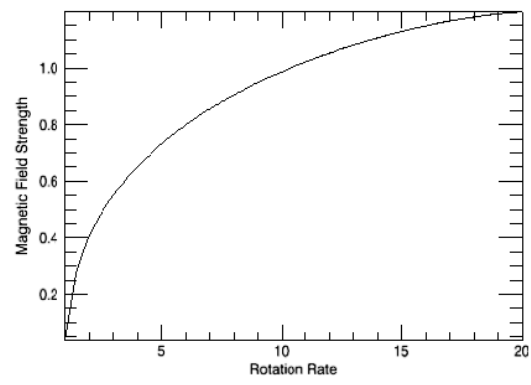

(b) $|B|$ as a function of $\Omega$

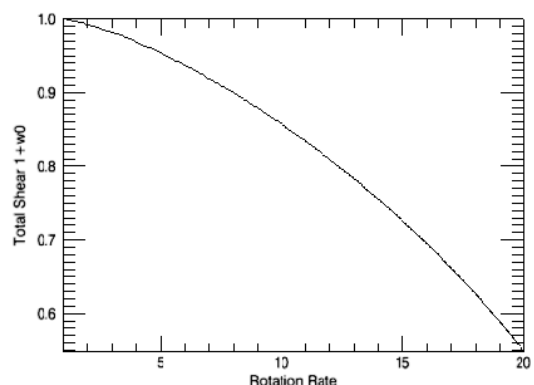

(c) Total shear as a function of $\Omega$

Fig. 6. $\omega_{\mathrm{M}},|B|$, and total shear are plotted as a function of $\Omega$ for $\kappa_{1}=\lambda_{1}=\lambda_{2}=3.5$ for Case 3 in the fifth-order system.

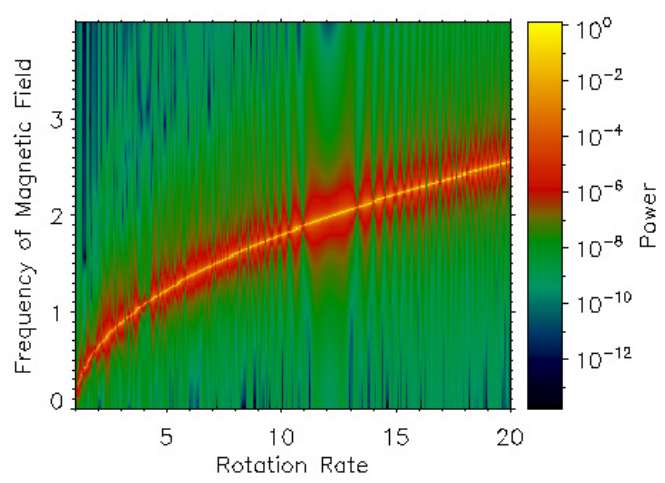

(a) $\omega_{M}$ as a function of $\Omega$

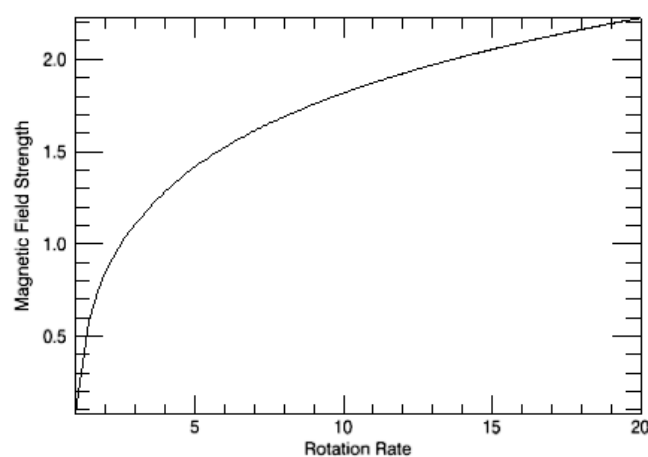

(b) $|B|$ as a function of $\Omega$

Fig. 7. Frequency of maximum intensity $\omega_{\mathrm{M}}$ and strength of magnetic field $|B|$ as a function of rotation rate $\Omega$ for $\kappa_{1}=\kappa_{2}=\lambda_{1}=\lambda_{2}=0.5$ for Case 1 in the sixth-order system.

Case 2: we consider the effect of shear quenching and loss due to magnetic flux in the absence of $\alpha$-quenching (i.e., $\kappa_{1}=0$, $\kappa_{2}=\lambda_{1}=\lambda_{2}=0.5$ ). Figure 8a shows the behavior of the frequency spectrum of magnetic field strength $B$ as a function of rotation rate. Frequency of maximum intensity $\omega_{\mathrm{M}}$, illustrated in yellow, increases with rotation rate. By using the power law $\omega_{\mathrm{M}}=\Omega^{\beta}$, we find the scaling of $\beta=1.17$, which is close to the observed range of $\beta=1.25 \pm 0.5$ (Noyes et al. 1984). In this case, we observe a red band of localized frequency around the maximum intensity frequency $\omega_{M}$, which broadens gradually with rotation rate due to dispersion. Moreover, a second branch of frequency of localized maximum is found above the main branch. This second branch is caused by the fluctuating differential rotation. In Fig. 8b, we study $|B|$ as a function of $\Omega$ and clearly see that the magnetic field strength increases very fast with rotation rate as compared to Case 1 . We find almost similar results for $\omega_{\mathrm{M}}$ and $|B|$ when we consider the effect of loss due to magnetic flux on dynamo action in the absence of $\alpha$ quenching and shear quenching, that is, for $\kappa_{1}=\kappa_{2}, \lambda_{1}=\lambda_{2} \neq 0$.

In summary, in all cases of the sixth-order system, the absence of mean differential rotation leads to a monotonic increase of magnetic field with rotation rate without saturation. That is, the severe quenching of dynamo and $w_{\circ}$ obtained in the fifth-order system in Sect. 4 is compensated by $w$, implying the complementary role of $w$ and $w_{\circ}$ in stellar dynamo and the necessity of incorporating both. Furthermore, frequency is found to be higher than that obtained in seventh-order system, confirming that $w_{\circ}$ and $w$ tend to generate lower and higher $\omega$, respectively.

\section{Minimal dynamical model: parameter dependencies}

In Sects. 2-5, we have shown that the best agreement with observations is obtained in Case 3 of the seventh-order system, which includes both quadratic $\alpha$-quenching and flux loss with the coefficients of order one. The success of Case 3 in reproducing observations is due to its capability of keeping a dynamical balance among the generation and dissipation of magnetic fields and also between the mean and fluctuating differential rotations. This balance makes the dynamo operate near the onset of dynamo instability, leading to an almost linear increase in frequency with rotation rate with a rather well-localized frequency (reminiscent of a linear dynamo), flattening of magnetic energy for high rotation, and quenching in total shear, consistent with observations. We demonstrate in this section that this seventh-order system is "minimal" in terms of the number of necessary parameters by investigating a more general seventh-order system, which has a different nonlinear power-law dependence of $\alpha$ quenching and flux loss on $B$ in Eqs. (7) and (8) and by checking on parameter dependencies. Generalized equations are given as follows:

$$
\begin{aligned}
\dot{A} & =\frac{2 D B}{1+\kappa_{1}\left(|B|^{m}\right)}-\left[1+\lambda_{1}\left(|B|^{n}\right)\right] A, \\
\dot{B} & =\mathrm{i}\left(1+w_{0}\right) A-\frac{1}{2} \mathrm{i} A^{*} w-\left[1+\lambda_{2}\left(|B|^{n}\right)\right] B, \\
\dot{w}_{0} & =\frac{1}{2} \mathrm{i}\left(A^{*} B-A B^{*}\right)-v_{0} w_{0}, \\
\dot{w} & =-\mathrm{i} A B-v w
\end{aligned}
$$




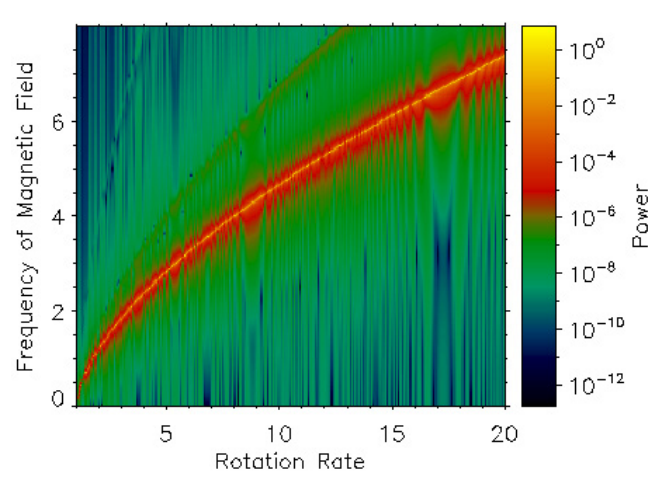

(a) $\omega_{M}$ as a function of $\Omega$

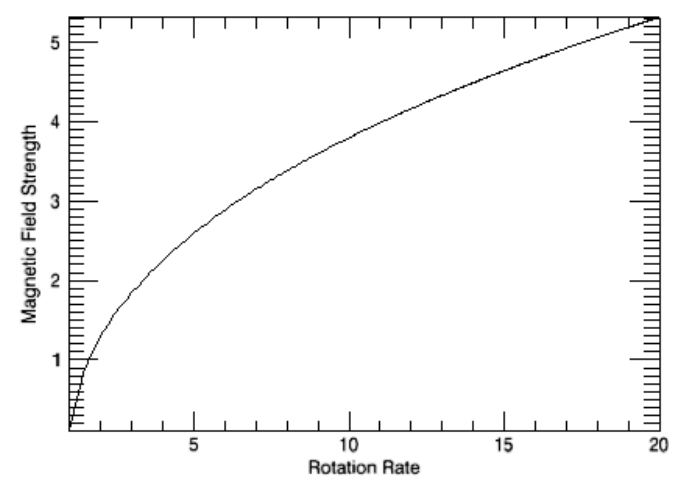

(b) $|B|$ as a function of $\Omega$

Fig. 8. Frequency of maximum intensity $\omega_{\mathrm{M}}$ and $|B|$ as a function of rotation rate $\Omega$ for $\kappa_{1}=0, \kappa_{2}=\lambda_{1}=\lambda_{2}=0.5$ for Case 2 in the sixth-order system.

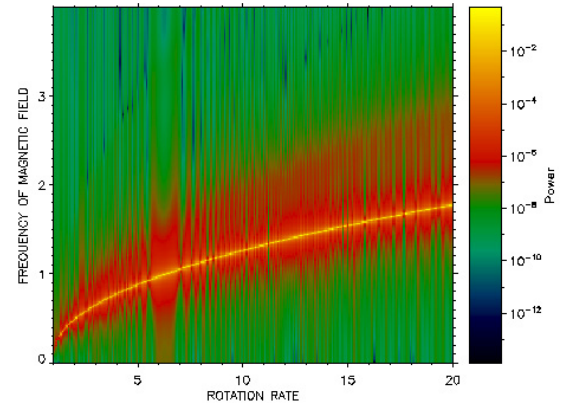

(a) $\omega_{\mathrm{M}}$ as a function of $\Omega$

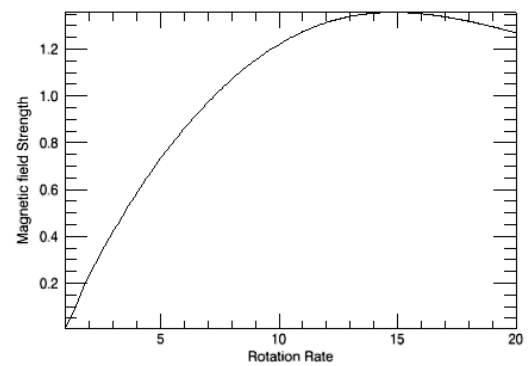

(b) $|B|$ as a function of $\Omega$

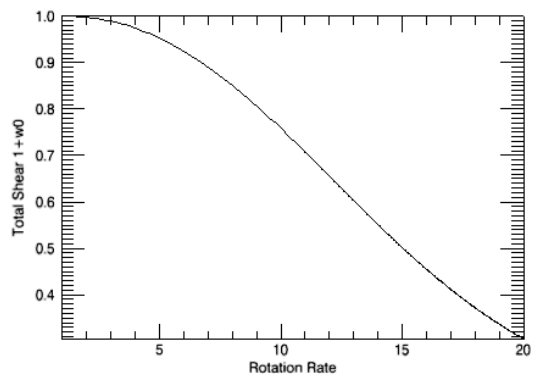

(c) Total shear as a function of $\Omega$

Fig. 9. Case 1: frequency of maximum intensity $\omega_{\mathrm{M}}$, magnetic field strength $|B|$, and total shear are plotted as a function of $\Omega$ for $m=n=1$ and $\kappa_{1}=\lambda_{1}=\lambda_{2}=2.5$.

where $m$ and $n$ are the power-law index of the $\alpha$-quenching term and the flux-loss terms, respectively. We have investigated about 35 different cases by varying systematically values of $m, n, \kappa_{1}, \lambda_{1}$ and $\lambda_{2}$. For all these cases and three cases in Sect. 3, we compared the main terms on the right hand side (RHS) of above Eq. (17) and also those in (18) (i.e., generation and dissipation terms); we confirmed that the observation is reproduced only when (i.e., Case 3 in Sect. 3) the $\alpha$-source term and magnetic dissipation are in balance in Eq. (17) with similar magnitude (within a factor of less than 2), while the generation of $A$ by shear and dissipation are in balance in Eq. (18). From this thorough investigation, we found that the $\alpha$-quenching power law and magnetic dissipation should increase at least quadratically ( $m \geq 2, n \geq 2$ ) for the coefficient of order 1 (i.e., $\left.0.5<\kappa_{1}, \lambda_{1}, \lambda_{2}<2.5\right)$. Among these cases, we present results for the following four cases as examples:

Case 1: $m=n=1$ and $\kappa_{1}=\lambda_{1}=\lambda_{2}=2.5$,

Case 2: $m=n=3$ and $\kappa_{1}=\lambda_{1}=\lambda_{2}=2.5$,

Case 3: $m=2, n=1$ and $\kappa_{1}=\lambda_{1}=\lambda_{2}=2.5$,

Case 4: $m=1, n=2$ and $\kappa_{1}=\lambda_{1}=\lambda_{2}=2.5$.

Case 1: dynamo action is ceased by the less efficient $\alpha$-quenching and flux loss due to magnetic field (i.e., $m=n=1$ and $\kappa_{1}=\lambda_{1}=\lambda_{2}=2.5$ ). In Fig. 9a, the frequency of maximum intensity $\omega_{\mathrm{M}}$ is plotted against rotation rate by depicting high to low intensity of frequency from yellow to dark black colors. Frequency of maximum intensity increases slowly with rotation rate, surrounded by a red band of lower frequency of maximum intensity that increases its width uniformly with increasing rotation rate.The dependence of $\omega_{\mathrm{M}}$ on $\Omega$ is found to be the power law as $\omega_{\mathrm{M}}=\Omega^{\beta}$ with scaling exponent of $\beta=0.80$ for stars with a rotation period of more than three days (i.e., $\Omega \leq 10)$ and $\beta=0.91$ for stars with a rotation period of less than three days (i.e., $\Omega<10$ ). These values lie within the observational limit of $\beta \sim 0.80$ and $\beta \sim 1.15$. However, the behavior of magnetic field strength $|B|$ with rotation rate shown in Fig. 9b does not seem to be compatible with observation. Specifically, $|B|$ increases with $\Omega$, attaining its maximum value around $\Omega \sim 15$ and then for $\Omega>16,|B|$ starts reducing slowly. This drop of $B$ is shown to be associated with shear quenching in Fig. 9c, which becomes too small for a high rotation rate. Therefore, inefficient magnetic dissipation through weaker quenching of alpha and flux loss leads to too strong magnetic fields. This reduces total shear, causing the decrease of $B$ for high rotation.

Case 2: saturation is caused by the stronger $\alpha$-quenching and flux loss due to the magnetic field (i.e., $m=n=3$ and $\left.\kappa_{1}=\lambda_{1}=\lambda_{2}=2.5\right)$. We now show that too strong alpha quenching and flux loss is also problematic, with the opposite effect. First, we study the behavior of frequency $\omega_{\mathrm{M}}$, illustrated in yellow for high intensity and with rotation rate $\Omega$ in Fig. 10a. We notice a slow increase in $\omega_{M}$ with rotation rate. A red band of localized frequency of maximum intensity is observed around $\omega_{\mathrm{M}}$ whose width is almost uniform. By using the power law, we investigate the dependence of $\omega_{\mathrm{M}}$ on $\Omega$ with scaling exponent $\beta$, 


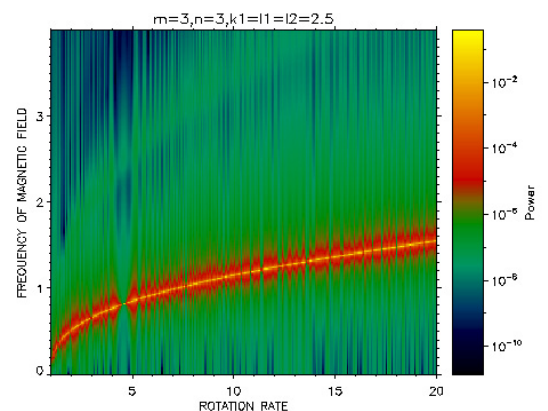

(a) $\omega_{\mathrm{M}}$ as a function of $\Omega$

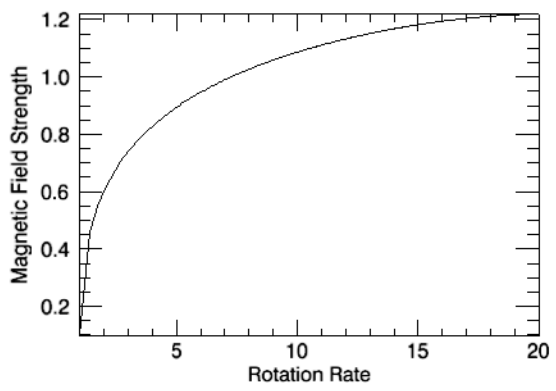

(b) $|B|$ as a function of $\Omega$

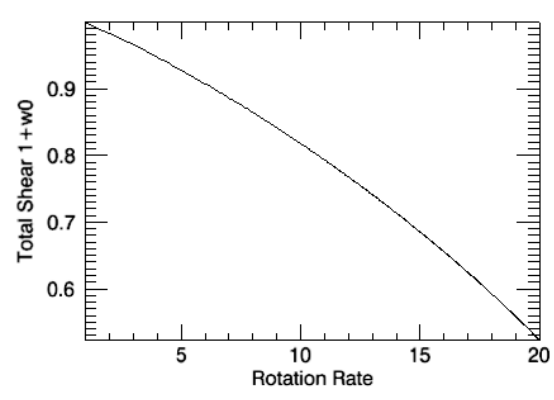

(c) Total shear as a function of $\Omega$

Fig. 10. Case 2: frequency of maximum intensity $\omega_{\mathrm{M}}$, magnetic field strength $|B|$, and total shear are plotted as a function of $\Omega$ for $m=n=3$ and $\kappa_{1}=\lambda_{1}=\lambda_{2}=2.5$.

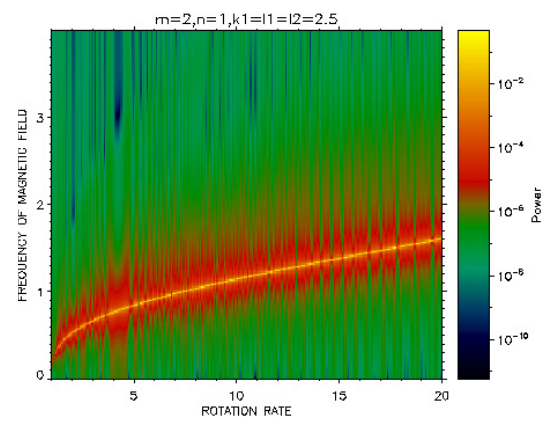

(a) $\omega_{\mathrm{M}}$ as a function of $\Omega$

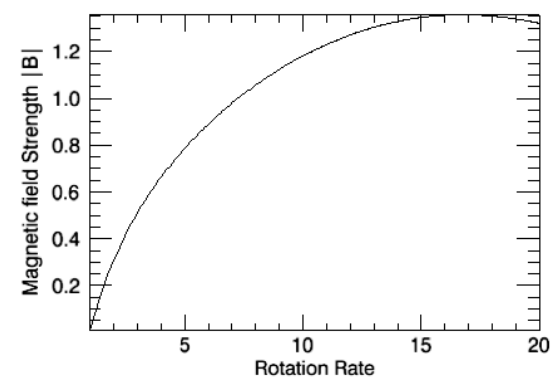

(b) $|B|$ as a function of $\Omega$

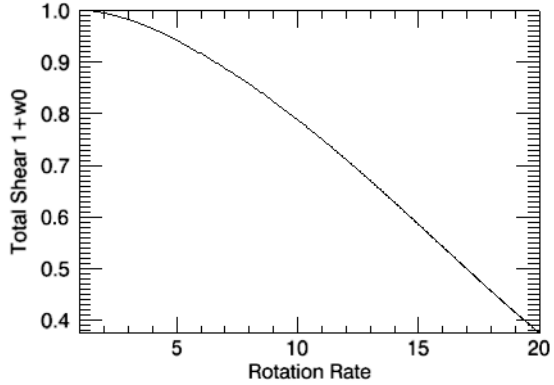

(c) Total shear as a function of $\Omega$

Fig. 11. Case 3: frequency of maximum intensity $\omega_{\mathrm{M}}$, magnetic field strength $|B|$, and total shear are plotted as a function of $\Omega$ for $m=2, n=1$ and $\kappa_{1}=\lambda_{1}=\lambda=2.5$.

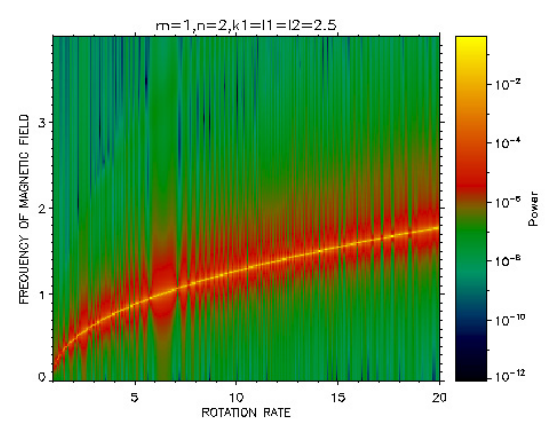

(a) $\omega_{\mathrm{M}}$ as a function of $\Omega$

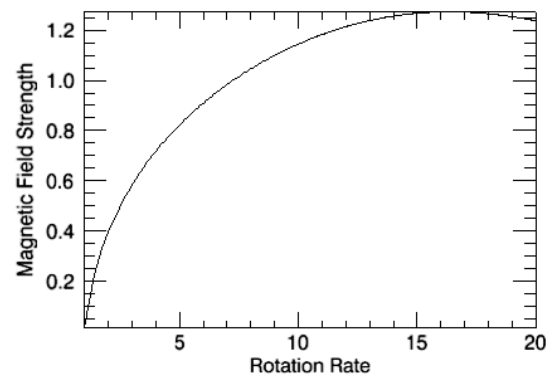

(b) $|B|$ as a function of $\Omega$

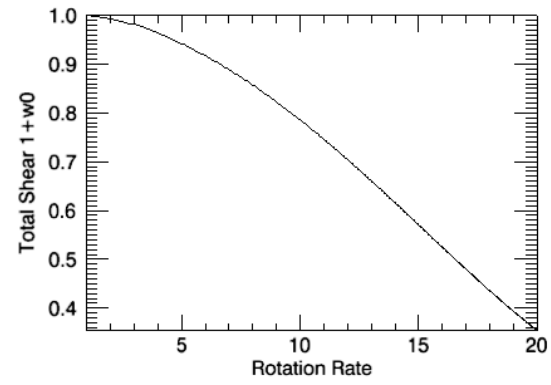

(c) Total shear as a function of $\Omega$

Fig. 12. Case 4: frequency of maximum intensity $\omega_{\mathrm{M}}$, magnetic field strength $|B|$, and total shear are plotted as a function of $\Omega$ for $m=1, n=2$, and $\kappa_{1}=\lambda_{1}=\lambda_{2}=2.5$.

whose value is found to be 0.75 in this case. This is very close to the observations. The strength of magnetic field $|B|$ is examined as a function of rotation rate in Fig. 10b. Clearly, $|B|$ is growing very slowly with rotation rate $\Omega$ without being saturated, correlated with the slow decline of total shear in Fig. 10c.

Case 3: we now consider the case where saturation is caused by quadratic $\alpha$-quenching, while the magnetic flux loss has a weaker, linear dependence on $B$ (i.e., $m=2, n=1$ and $\left.\kappa_{1}=\lambda_{1}=\lambda_{2}=2.5\right)$. In Fig. 11a, a well-defined frequency of maximum intensity $\omega_{M}$ is shown as a function of rotation rate, depicting high to low frequency in yellow to dark black. The $\omega_{M}$ increases very slowly with rotation rate and a very broad band of localized frequency of maximum intensity is noticed around $\omega_{\mathrm{M}}$, which widens gradually with $\Omega$. Power-law scaling $\beta$ of frequency of maximum intensity with rotation rate is consistent with observations for stars with a rotation period of less than two days $(\Omega>15)$ with $\beta=0.94$, whereas for stars with a rotation period of more than two days $(\Omega<15)$, we find $\beta=0.73$, which is close to observations. The magnetic field strength $|B|$ grows gradually with the rotation rate and acquires its maximum value of 1.35 for $\Omega=17$. As $\Omega$ becomes more than 17 , we notice a slow decrease in $|B|$ (cf. Fig. 11b). Total shear is found to be reduced with $\Omega$ in Fig. 11c and responsible for the decrease in $|B|$.

Case 4: dynamo action is saturated by weaker $\alpha$-quenching and stronger magnetic flux losses i.e., $m=1, n=2$ and $\kappa_{1}=\lambda_{1}=$ $\lambda_{2}=2.5$. As shown in Fig. 12a, a well-defined frequency of maximum intensity $\omega_{\mathrm{M}}$, represented in yellow, increases faster with $\Omega$ than in previous case (cf. Fig. 11a). In contrast, the red band of lower frequency of maximum intensity around $\omega_{M}$ consistently broadens its width with rotation rate. In this case the power-law exponent $\beta$ possesses a value of 0.88 , which is 
in agreement with observations. However, the strength of magnetic field $|B|$ increases with $\Omega$ reaching a maximum value of $|B|=1.27$ around $\Omega \sim 17$ and starts decreasing with increasing $\Omega>17$ as noted in Fig. 12c. This is incompatible with observations. Shear quenching due to the Lorentz force, which causes the decrease in $|B|$ shown in Fig. 12c declines slowly with $\Omega$.

For the four cases discussed above and all other cases we have examined, we compared the main terms on the RHS of Eq. (17) and also those in (18) (i.e., generation and dissipation terms). We confirmed that disagreement between the results of these cases and the observation is associated with imbalance between the $\alpha$-source term and the magnetic dissipation in Eq. (17) and/or between the generation of $A$ by shear and dissipation in Eq. (18). In-depth discussion on this balance and on the effect of various different terms in our model is presented in Sood \& Kim (2013) by using analytical and numerical simulations. In summary, our seventh-order system in Sect. 2 seems to be a minimal model that ensures a right balance between all the nonlinearities, such as $\alpha$-quenching and flux losses due to magnetic field and their respective coefficients, which gives results consistent with observations.

\section{Conclusions}

We have proposed a minimal dynamical model of dynamo and differential rotation given by seventh-order system by including the effect of $\alpha$-quenching, magnetic flux loss, and the Lorentz force on mean $w_{\circ}$ and fluctuating differential $w$ rotations. By varying the degree of $\alpha$-quenching and magnetic flux loss, we examined how magnetic field strength, frequency (with $\omega_{M}$ at maximum intensity), and differential rotation vary with rotation rate $\Omega$. Our results show a good agreement with observations when $\alpha$-quenching and loss due to magnetic flux are taken into account to a similar degree with a quadratic dependence on $B$. Specifically, in this case, we obtained almost linear increase in frequency with rotation rate with a rather well-localized frequency (reminiscent of a linear dynamo), flattening of magnetic energy for high rotation, and quenching in total shear. This is consistent with observations. Detailed analysis of this (and a more general) seventh-order system and its limiting cases of fifth- and sixth-order systems suggest that a right balance between $w_{\circ}$ and $w$ is necessary to obtain $\omega_{\mathrm{M}}$ consistent with observations. This implies that both upward $\left(w_{\circ}\right)$ and downward $(w)$ scale coupling to the differential rotation is important for the dynamo process.

Interestingly, this balance seems to be indicative of a collective, organized process in stellar dynamo, which operates near marginal stability as a result of balance not only between the generation and destruction of magnetic fields but also between poloidal and toroidal magnetic fields and between mean and fluctuating differential rotation. That is, in contrast to the traditional view, stellar dynamos could be a result of a self-organizing process where a long range correlation in stellar interiors and open boundary is important. Furthermore, an almost linear relation between observed magnetic frequency and rotation rate could be another signature of self-organization, with the dynamo working near stability. In this sense, it is not coincidental that various instability mechanisms involved in dynamos, the effect of meriodinal circulation, and magnetic flux loss have received great attention in dynamos in recent years (e.g., see Charbonneau 2005; Jouve et al. 2010; Miesch \& Brown 2012). Investigations into necessary balance in dynamical models and its precise physical mechanism in the context of stellar rotation evolution are in progress and will be addressed in future publications (Sood \& Kim 2013).

Finally, we note that while our dynamical model is limited, especially, because it is incapable of capturing the spatial evolution of stellar magnetic fields, it seems to have certain merit of being less sensitive to the precise form of the dynamo. The $\alpha$-effect and flux losses are simple parameterizations of the nonlinear generation and destruction of magnetic fields in stars. Furthermore, a long-range correlation seems to be conveniently incorporated in the evolution of low-harmonic modes in our model. Thus, despite its simplicity, it can serve as a useful model that can explain observed frequency and strength of magnetic field and the differential rotation for stars with different rotation rates. The usefulness of a dynamical model was recently demonstrated in the context of shear flow dynamics (e.g., self-organization) where a reduced dynamical model successfully reproduced the essential characteristics of flows obtained in full 2D hydrodynamic turbulence (Newton \& Kim 2012). Future work will investigate the extension of our model to include other physical effects, especially stochasticity in the parameters in dynamo model (e.g., stochastic $\alpha$ effect) (Newton \& Kim 2012; Proctor 2007; Richardson \& Proctor 2010). We note that the signature of self-organization of solar dynamos was recently reported in a stochastic dynamo (Newton \& Kim 2013).

Acknowledgements. We thank B. Brown for the helpful discussion.

\section{References}

Barnes, J. R., Collier Cameron, A., Donati, J.-F., et al. 2005, MNRAS, 357, L1 Cattaneo, F., Jones, C. A., \& Weiss, N. O. 1983, IAU Symp., 102, 307

Charbonneau, P. 2005, Liv. Rev. Sol. Phys., 2, 2

(http: www . livingreviews .org/lrsp-2005-2)

Charbonneau, P., \& Saar, S. H. 2001, in Magnetic Fields across the H-R digram, eds. G. Mathys, S. K. Solanki, \& D. T. Wickamasinghe, ASP Conf. Ser., 248, 189

Dikpati, M., \& Charbonneau, P. 1999, ApJ, 518, 508

Donahue, R. A., Saar, S. H., \& Baliunas, S. L. 1996, ApJ, 466, 384

Fröhlich, H.-E., Küker, M., Hatzes, A. P., \& Strassmeier, K. G. 2009, A\&A, 506, 263

Gilman, P. A. 1983a, IAU Symp., 102, 247

Gilman, P. A. 1983b, ApJS, 53, 243

Ivanova, T. S., \& Ruzmaikin, A. A. 1977, Sov. Astron., 21, 479

Jepps, S. A. 1975, J. Fluid Mech., 67, 625

Jouve, L., Brown, B. P., \& Brun, A. S. 2010, A\&A, 509, A32

Kitchatinov, L. L., \& Olemskoy, S. V. 2011, MNRAS, 411, 1059

Miesch, M. S., \& Brown, B. P. 2012, ApJ, 746, L26

Moffatt, H. K. 1978, Magnetic Field Generation in Electrically Conducting Fluids (Cambridge: Cambridge University Press)

Newton, A. P. L., \& Kim, E. 2012, Phys. Plasmas, 19, 7, 072310

Newton, A. P. L., \& Kim, E. 2013, A\&A, 551, A66

Noyes, R. W., Weiss, N. O., \& Vaughan, A. H. 1984, ApJ, 287, 769

Parker, E. N. 1979, Cosmical Magnetic Fields (Oxford: Clarendon Press)

Proctor, M. R. E. 2007, MNRAS, 382, L39

Reiners, A., \& Schmitt, J. 2003, A\&A, 398, 647

Rempel, M. 2006, ApJ, 647, 662

Richardson, K. J., \& Proctor, M. R. E. 2010, GAFD, 104, 5

Robinson, R. D., \& Durney, B. R. 1982, A\&A, 108, 322

Saar, S. H. 2002, in the 11th Cool Stars: Stellar System and the Sun, eds. R. J.

Garcia Lopez, R. Rebolo, \& M. R. Zapatero, ASP Conf. Ser., 223, 292

Saar, S. H. 2011, International Astronomical Union

Saar, S. H., \& Brandenburg, A. 1999, ApJ, 524, 295

Sood, A., \& Kim, E. 2013, A\&A, submitted

Weiss, N. O., Cattaneo, F., \& Jones, C. A. 1984, Geophys. Astrophys. Fluid Dyn., 30, 305

Yoshimura, H. 1975, ApJS, 29, 467

Yoshimura, H. 1978, ApJ, 220, 692 